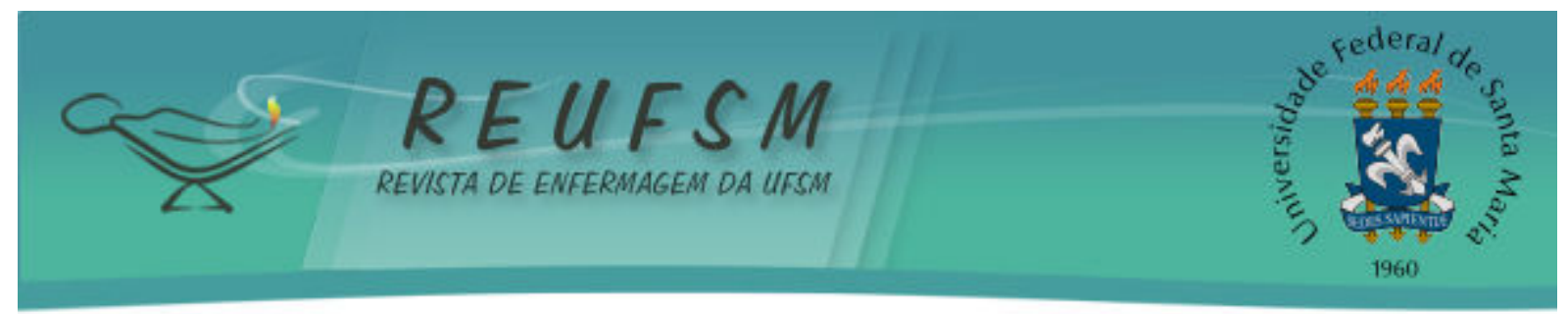

ARTIGO ORIGINAL

\title{
CONSULTA DE ENFERMAGEM EM PUERICULTURA: UM ENFOQUE NOS REGISTROS DE ATENDIMENTOS
}

NURSE CONSULTATION IN CHILD CARE: A FOCUS ON MEDICAL RECORDS

CONSULTA DE ENFERMERÍA EN CUIDADO DEL NIÑO: UN ENFOQUE EN LOS REGISTROS MÉDICOS

\author{
Tatiane Baratieri ${ }^{1}$ \\ Leticia Gramazio Soares ${ }^{2}$ \\ Maria Luciana Botti ${ }^{3}$ \\ Araceli Conrado Campanini ${ }^{4}$
}

Doi: $10.5902 / 217976928553$

RESUMO: Objetivo: analisar a puericultura realizada pelo enfermeiro, apontando as potencialidades e limitações. Método: estudo documental, descritivo-exploratório, quantitativo, com coleta de dados em fevereiro de 2012 junto a 46 prontuários de crianças atendidas pelo enfermeiro em consulta de puericultura, em uma unidade de saúde de um município do centro-sul paranaense; os dados foram analisados por estatística descritiva. Resultados: apenas $17 \%$ das crianças iniciaram a puericultura no primeiro mês de vida; $32 \%$ foram atendidas com frequência irregular; quanto aos dados registrados no prontuário, $100 \%$ tinham registro de peso; $89 \%$ de estatura e perímetro cefálico; $98 \%$ tipo de alimentação; apenas um havia registro do desenvolvimento neuropsicomotor; $100 \%$ não possuíam registro de perímetro torácico e abdominal, e vacinação. Conclusão: a puericultura tem como potencialidades promover a saúde e prevenir agravos às crianças. Como limitações, evidenciou-se atendimento pautado na demanda espontânea e no curativismo, falta de registros e análise dos dados coletados, comprometendo a qualidade da puericultura.

Descritores: Saúde da criança; Atenção primária à saúde; Enfermagem.

ABSTRACT: Aim: to analyze the child care provided by nurses, identifying strengths and limitations. Method: documentary, descriptive-exploratory and quantitative study, with data collection in February 2012 with 46 medical records of children seen by the nurse in child care consultation in a health center of a city in Paraná; the data were analyzed by descriptive statistics. Results: only $17 \%$ of children initiated the child care in their first month of life; 32\% were seen with irregular frequency; concerning the data recorded in the medical record, $100 \%$ had weight record; $89 \%$ height and head circumference records; 98\% type of food; only one had record of neuropsychomotor development; $100 \%$ did not have record of thoracic and abdominal circumference, and vaccination. Conclusions: the child care has the potential to promote health and prevent injuries to children. As limitations, it was noticed the care based on spontaneous demand and curativism, lack of records and analysis of collected data, compromising the quality of child care.

Descriptors: Child health; Primary health care; Nursing.

RESUMEN: Objetivo: analizar la puericultura realizada por el enfermero, identificando potencialidades y limitaciones. Método: estudio documental, exploratorio y descriptivo,

\footnotetext{
${ }^{1}$ Enfermeira. Mestre. Docente do curso de Enfermagem da Universidade Estadual do Centro-Oeste - UNICENTRO. Guarapuava/PR/Brasil. E-mail: baratieri.tatiane@gmail.com

2 Enfermeira. Mestre. Docente do curso de Enfermagem da UNICENTRO. E-mail: leticiagramazio13@gmail.com

${ }^{3}$ Enfermeira. Mestre. Docente do curso de Enfermagem da UNICENTRO. E-mail: malubotti@yahoo.com.br

${ }^{4}$ Enfermeira. Especialista em Gestão em Saúde. Enfermeira da Secretaria Municipal de Saúde de Palmital/PR. E-mail: araceliconrado@yahoo.com.br
} 


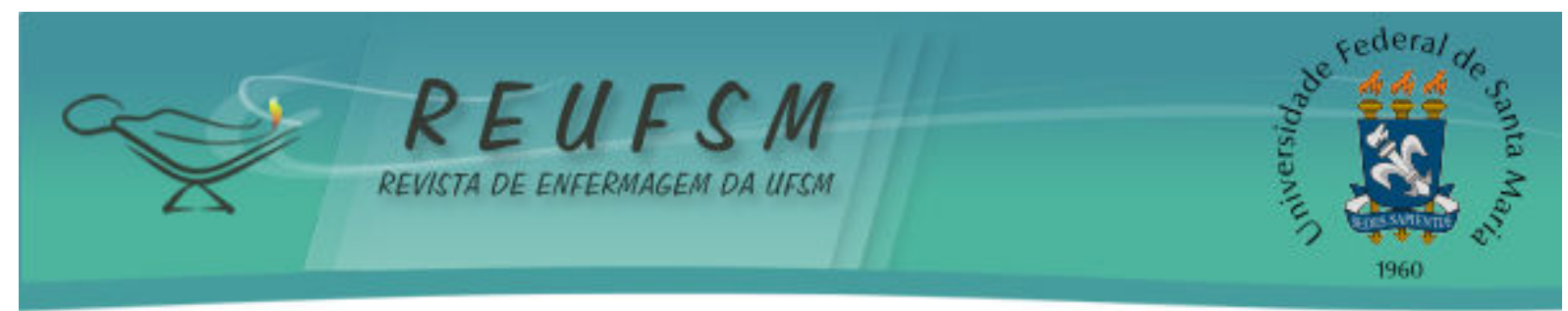

cuantitativo, con recolección de datos en febrero de 2012 en 46 registros médicos atendidos en consulta de puericultura, en un centro de salud de Paraná (Brasil); los datos fueron analizados por estadística descriptiva. Resultados: sólo $17 \%$ de los niños iniciaron puericultura al primer mes de vida; 32\% fueron atendidos con frecuencia irregular; sobre datos del registro médico, 100\% tuvieron registro de peso; $89 \%$ de estatura y perímetro cefálico; $98 \%$ del tipo de alimentación; sólo uno tenía registro del desarrollo neuropsicomotor; $100 \%$ no tenían registrado perímetro torácico o abdominal, ni vacunación. Conclusiones: la puericultura tiene como potencialidades promover la salud y prevenir lesiones a los niños. Como limitaciones, se observó el cuidado basado en demanda espontánea y curativismo, y la falta de registros y análisis de datos recogidos, comprometiendo la calidad de la puericultura.

Descriptores: Salud del niño; Atención primaria de salud; Enfermería.

\section{INTRODUÇÃO}

A puericultura é a ciência que engloba conhecimentos básicos de fisiologia, higiene, nutrição, sociologia, cultura, desenvolvimento e comportamento que viabilizam o desenvolvimento neuropsicomotor (DNPM) das crianças. Nesse âmbito, o cuidado prestado em puericultura visa à promoção da saúde e à prevenção de agravos, impactando em melhor qualidade de vida tanto para a criança, como para a família. ${ }^{1}$

o Programa de Puericultura tem como propósito acompanhar o crescimento e o desenvolvimento infantil, observar a cobertura vacinal, estimular o aleitamento materno, orientar a introdução da alimentação complementar e prevenir as doenças que mais frequentemente acometem as crianças no primeiro ano de vida, como a diarreia e as infecções respiratórias. ${ }^{2}$

Para garantir a saúde integral da criança é necessária a postura ativa dos profissionais de saúde, com uma agenda de compromissos a serem cumpridos para a promoção do bem-estar infantil. A vigilância da saúde da criança inclui a puericultura, a imunização, a identificação de violências e abusos, a detecção e monitoramento de doenças crônicas, a promoção da saúde e de hábitos saudáveis. Portanto, é de fundamental importância um programa de puericultura efetivamente implantado e que atue em rede com os profissionais de referência. ${ }^{3-4}$

A puericultura é considerada uma importante estratégia de cuidado preventivo, sendo um dos eixos das ações desenvolvidas na atenção à saúde da criança. É capaz de orientar e promover ações de promoção da saúde e de bem-estar infantil, além de permitir a detecção precoce de problemas de saúde e oportunizar seu tratamento em tempo hábil. Esse seguimento prevê um calendário básico de consultas que deve ser cumprido corretamente, promovendo a busca ativa dos faltosos. ${ }^{5}$ Para organizar o acompanhamento do crescimento e desenvolvimento infantil, o Ministério da Saúde prevê que toda criança deve passar por, no mínimo, sete consultas de puericultura no primeiro ano de vida. ${ }^{6}$

Toda criança deve receber a Caderneta de Saúde da Criança, de preferência ainda na maternidade, na qual são registrados os dados de identificação da criança, da história obstétrica e neonatal, da alimentação, do crescimento e desenvolvimento, da saúde bucal, auditiva e visual, das vacinações, além do registro das intercorrências clínicas. Contém, ainda, orientações para a promoção da saúde e prevenção de agravos.

A utilização da Caderneta permite o registro correto e completo das informações, sendo considerada requisito básico para vigilância e promoção da saúde infantil; o diálogo com a família sobre as anotações realizadas, bem como o registro dos dados antropométricos nos gráficos avaliativos de crescimento; a observação do desenvolvimento neuropsicomotor, de acordo com tabela padronizada do desenvolvimento infantil conforme a idade da criança e; os registros do esquema vacinal. 


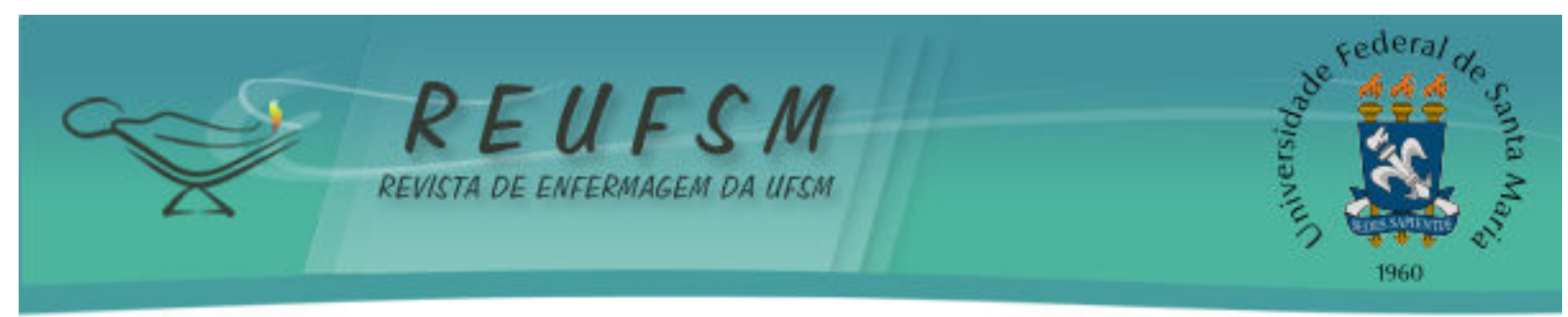

O enfermeiro é fundamental na realização da puericultura, pois esta envolve uma sequência de etapas que direcionam as ações de modo que haja um atendimento eficaz às necessidades da saúde da criança e aos anseios da família. Não se trata apenas de aferir as medidas antropométricas, mas sim avaliar a criança na sua integralidade, observando crescimento e DNPM, com ênfase nas orientações de cuidado. Vale salientar que a puericultura se constitui em um elemento indispensável ao processo de trabalho do enfermeiro. ${ }^{4,7}$

A consulta de enfermagem em puericultura deve ser vista como estratégia de promoção da saúde por meio de ações educativas, que consistem em avaliar e promover a aquisição de competências para atender também a outras necessidades das crianças, tais como comunicação, higiene, imunização, sono, nutrição, afeto, amor, solicitude e segurança. Sendo de grande relevância que haja orientação eficaz para as mães no que diz respeito ao cuidado com seus filhos. ${ }^{8}$

Este estudo se justifica pela necessidade de se conhecer como acontece a consulta de enfermagem em puericultura, tendo em vista que acompanhar o crescimento e o desenvolvimento é o eixo central e integrador de todas as ações de saúde da criança por meio do atendimento adequado das necessidades de saúde infantil, nas diferentes dimensões, biológicas e/ou socioculturais.

É relevante conhecer a realidade da puericultura, pois essa é uma estratégia de promoção da saúde e prevenção a agravos, voltada para vigilância da saúde infantil, a qual interfere positivamente na redução da morbimortalidade dessa população. Destarte, definiu-se como questão norteadora para o estudo: A partir dos registros do enfermeiro, quais são as potencialidades e os limites da consulta de enfermagem em puericultura?

Tendo em vista a importância do acompanhamento do crescimento e desenvolvimento infantil, objetivou-se com este estudo analisar a puericultura realizada pelo enfermeiro, apontando as potencialidades e limitações.

\section{MÉTODO}

Trata-se de um estudo documental, descritivo-exploratório, com abordagem quantitativa. O local de estudo foi uma Unidade Básica de Saúde que atua nos moldes da Estratégia da Saúde da Família (ESF), em município do centro-sul paranaense.

A opção metodológica se deu porque o evento já ocorreu e os dados estão contidos nos prontuários das crianças que realizaram puericultura na unidade estudada. Nesse método de pesquisa documental, a mensuração se dá após a ocorrência dos fatos no espaço e no tempo, e os dados são encontrados nos registros da história vivida por tais crianças. ${ }^{9}$

A população estudada foi composta por todos os prontuários de crianças de 0 a 1 ano, que passaram por pelo menos um atendimento de puericultura realizado por profissional enfermeiro, entre os meses de novembro de 2010 e novembro de 2011, o que resultou em 46 prontuários.

A coleta dos dados foi realizada no mês de fevereiro de 2012 e norteada por um roteiro elaborado pelos pesquisadores. A presente pesquisa analisou pontos específicos relacionados às puericulturas realizadas pelos enfermeiros na ESF estudada. Sendo assim, verificaram-se os seguintes itens: a faixa etária das crianças ao iniciarem as consultas, a frequência do acompanhamento e as ações realizadas pelo enfermeiro durante a puericultura.

Após a coleta, os dados foram analisados por estatística descritiva, sendo estes apresentados em tabelas e discutidos com base na literatura disponível sobre o tema.

A pesquisa se respalda na Resolução 196/1996 sendo, portanto, encaminhada ao Comitê de Ética e Pesquisa da Universidade Estadual do Centro Oeste/UNICENTRO, para avaliação e aprovação. Por se tratar de uma pesquisa documental, com dados coletados de 


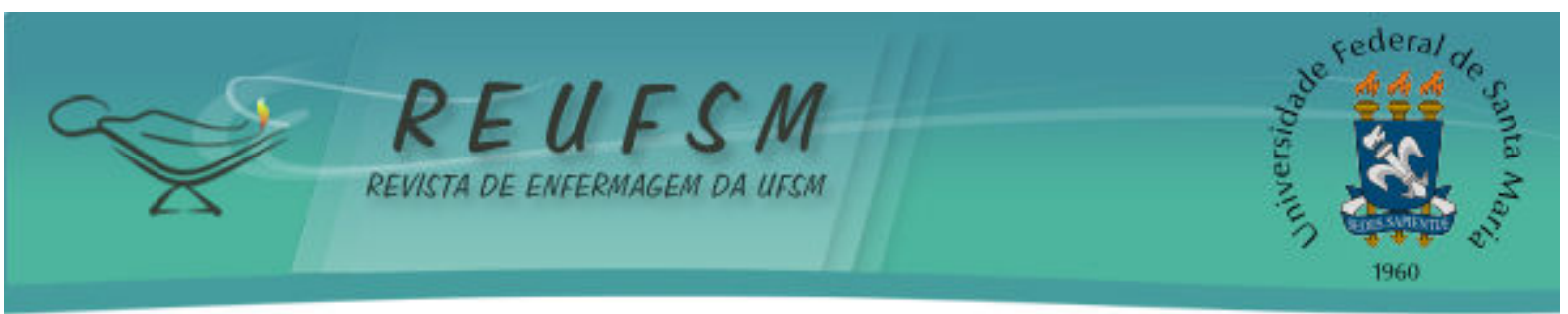

prontuários, solicitou-se ao Comitê de Ética a dispensa da utilização do Termo de Consentimento Livre e Esclarecido. Ressalta-se que o anonimato dos usuários, bem como demais informações das famílias, foram preservados e, também, que a pesquisa foi autorizada por intermédio do parecer do ofício $\mathrm{n}^{\circ}$ 010/2012.

\section{RESULTADOS E DISCUSSÃO}

Foram identificados, no período proposto pela pesquisa, $46(100 \%)$ atendimentos de crianças registradas no Programa de Puericultura e acompanhadas pelo enfermeiro no decorrer do primeiro ano de vida, na unidade em estudo.

A Tabela 1 apresenta a faixa etária das crianças ao iniciarem a puericultura. Foi possível verificar que, dos 46 prontuários investigados, apenas oito crianças (17\%) iniciaram o acompanhamento de puericultura com um mês de vida, havendo um número elevado de crianças iniciando tardiamente.

Tabela 1: Distribuição da faixa etária de início do acompanhamento de puericultura. Palmital/PR, 2012. $N=46$

\begin{tabular}{ccc}
\hline FAIXA ETÁRIA EM MESES & N & $\%$ \\
\hline 1 & 8 & 17 \\
2 & 10 & 22 \\
3 & 9 & 20 \\
4 & 8 & 17 \\
5 & 6 & 13 \\
6 & 3 & 7 \\
11 & 1 & 2 \\
13 & 1 & 2 \\
\hline
\end{tabular}

A Tabela 1 demonstra que, apesar de a maioria dos atendimentos de puericultura iniciarem nos primeiros meses, eles estão fora do padrão estabelecido pelo Ministério da Saúde $^{6}$, no qual a primeira consulta precisa ocorrer antes de completar um mês, preconizando-se que se realize em até 15 dias de vida da criança. Nesse período, o enfermeiro pode orientar a mãe quanto aos cuidados ao recém-nascido, entre eles destaca-se o aleitamento materno, o controle do ganho de peso, o cuidado com o coto umbilical, a vacinação e as orientações gerais de cuidado e higiene. ${ }^{6}$

Outra importância da consulta precoce recai sobre o fato de que, no Brasil, a mortalidade neonatal (óbitos ocorridos até o $27^{\circ}$ dia de vida) é responsável por aproximadamente $70 \%$ de todas as mortes de crianças até o primeiro ano de vida. ${ }^{10}$ Diante desse fato, é possível relacionar o alto índice com cuidados prestados pelos profissionais de saúde, não somente durante o período de gestação e nascimento, mas inclusive no acompanhamento nos primeiros dias de vida em domicílio, pois muitas causas de óbitos neonatais podem ser evitadas pelo acompanhamento sistematizado com as unidades de saúde.

O contato precoce do enfermeiro com mãe e filho pode prevenir muitas intercorrências, visto que ambos estão em um processo de adaptação, no qual mudanças biológicas, sociais e emocionais estão ocorrendo. Portanto, dúvidas, medos e sensações diferentes permeiam essa fase, logo o enfermeiro pode assisti-los, fornecendo orientações e encaminhamentos adequados às necessidades de saúde desses sujeitos. Além disso, o seguimento da criança também estreita e fortalece o vínculo da família com a unidade de saúde. 


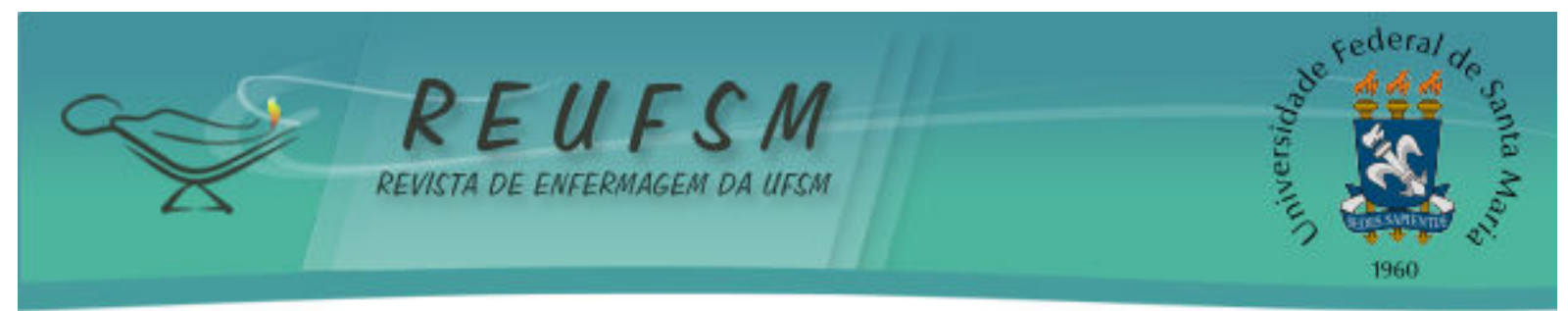

Pelo acompanhamento do crescimento e desenvolvimento, é possível estabelecer condutas preventivas adequadas à idade sobre vacinação, alimentação, estimulação e cuidados gerais com a criança, em um processo contínuo de educação em saúde. ${ }^{6}$ Contudo, o início tardio da puericultura, tal qual se observa na ESF estudada, pode influenciar negativamente nesse processo, interferindo na detecção precoce de doenças e agravos da infância, o que não permite encaminhamentos e resolução de problemas, muitas vezes simples, mas que terão repercussão na qualidade de vida das crianças. Para tanto, é necessário esforço da equipe de saúde para que desde o pré-natal as mães já sejam sensibilizadas e preparadas para iniciarem a puericultura antes dos 15 dias de vida da criança, para assim garantirem crescimento e desenvolvimento sadio a seus filhos.

Apesar das mudanças ocorridas na orientação e necessidades de atenção por meio do sistema de saúde atual, a literatura aponta que a realização da puericultura pelo enfermeiro ainda tem a organização do seu processo de trabalho centrada em procedimentos, com oferta de assistência baseada na doença, não sendo priorizadas as medidas preventivas. ${ }^{4}$

Tal situação confere ao serviço uma característica de atendimento à necessidade iminente, ou seja, por demanda espontânea, tendo como ponto de partida o agravo, sendo a assistência centrada na doença e cura, em detrimento a um modelo de atenção que promova a saúde.

Após a primeira consulta, as demais devem ocorrer com um mês, dois, quatro, seis, nove e 12 meses, totalizando, assim, sete consultas no primeiro ano de vida da criança. ${ }^{6}$ A Tabela 2 apresenta a frequência em que as consultas de puericultura foram realizadas no local estudado.

Tabela 2: Distribuição da frequência de acompanhamento de puericultura de crianças menores de um ano. Palmital/PR, 2012. $\mathrm{N}=46$

\begin{tabular}{ccc}
\hline FREQUÊNCIA & N & $\%$ \\
\hline Semanal & 3 & 7 \\
Quinzenal & 4 & 9 \\
Mensal & 22 & 48 \\
Bimestral & 2 & 4 \\
\hline
\end{tabular}

Para pesquisar a frequência que as crianças foram levadas à unidade para consulta de puericultura, não foi considerada a faixa etária destas. Utilizou-se como critério o que preconiza o Ministério da Saúde, já discutido anteriormente, ou seja, o ideal seria que as crianças de zero a quatro meses sejam atendidas mensalmente, após com seis, nove e 12 meses. ${ }^{6}$ Dessa forma, mesmo sem cruzar este dado em discussão com a faixa etária das crianças, nota-se que a frequência de atendimento não está de acordo com as normas do Ministério da Saúde, pois é possível perceber atendimentos irregulares.

Do total de 46 prontuários analisados, observou-se que 22 (48\%) realizaram puericultura mensalmente. Porém, 15 (32\%) das crianças acompanhadas fizeram puericultura em períodos irregulares. Sobre a frequência e o espaçamento nos atendimentos, verificou-se que ocorrem semanal, quinzenal e bimestralmente e é importante considerar que devem ser definidos pelo enfermeiro, considerando os riscos biológicos e sociais que a criança e a família apresentam. Várias condições, de distintas dimensões, como baixo peso ao nascer, prematuridade, malformações, dificuldade para amamentação, situação socioeconômica e de higiene precárias, e até o preparo da mãe para cuidar do recém-nascido, podem conferir à puericultura frequência maior do que é preconizado pelo Ministério da Saúde. 


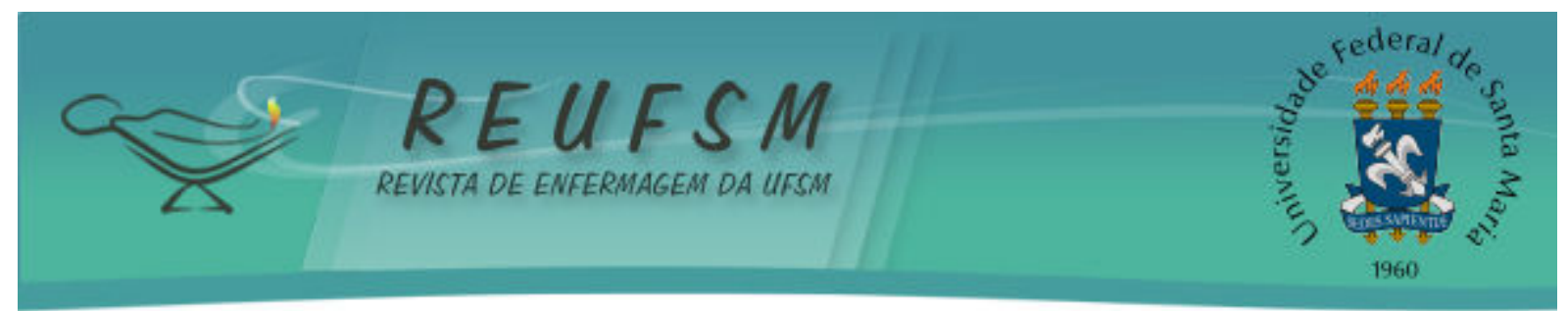

A detecção dos casos de crianças em risco para adoecer ou desenvolver comprometimentos na saúde é imprescindível, pois contribui para minimizar futuras complicações de saúde. ${ }^{11}$ No entanto, o enfermeiro deve ter preparo, competência, conhecimento e habilidade técnica para detectar as crianças de risco, as quais necessitarão de acompanhamento mais frequente.

A frequência em que o enfermeiro acompanha a criança é fator decisivo no crescimento e desenvolvimento infantil. A consulta de enfermagem visa a prestar assistência integral, resolutiva, contínua e de boa qualidade para a população, dentre as várias ações que priorizam promover a saúde, prevenir e curar as doenças, contribuindo ainda para que essa criança seja um adulto sadio e pleno no que se refere à possibilidade de alcançar a qualidade de vida. ${ }^{11}$

A frequência de acompanhamento do crescimento e desenvolvimento da população infantil estudada pode ser um indicador da qualidade no atendimento à criança. O Ministério da Saúde afirma que o calendário e o acompanhamento sistemático do crescimento e desenvolvimento caracterizam indicativos da qualidade da atenção prestada à criança. ${ }^{6}$

Vale ressaltar que na unidade pesquisada, no que tange ao atendimento de puericultura, identificou-se a inexistência de protocolos de atendimento e, também, a falta de um fluxograma de encaminhamento das crianças para o atendimento com o enfermeiro responsável. Salienta-se que isso pode, de alguma forma, explicar a inadequação na frequência de atendimento às crianças, demonstrando que a atenção é organizada conforme a demanda espontânea.

Outro dado pesquisado neste estudo se refere aos registros antropométricos como peso, estatura, perímetro cefálico $(\mathrm{PC})$, perímetro torácico $(\mathrm{PT})$, perímetro abdominal (PA), registros sobre DNPM, tipo de alimentação, imunização e se houve orientações registradas às mães ou responsáveis. Tais dados podem ser visualizados na Tabela 3.

Tabela 3: Informações referentes à consulta de enfermagem em puericultura no prontuário de crianças menores de um ano. Palmital/PR, 2012. $\mathrm{N}=46$

\begin{tabular}{lcccc}
\hline \multirow{2}{*}{ Informações registradas no prontuário } & \multicolumn{2}{c}{ Sim } & \multicolumn{2}{c}{ Não } \\
\cline { 2 - 6 } Peso & $\mathbf{N}$ & $\%$ & $\mathbf{N}$ & $\%$ \\
Tipo de alimentação & 46 & 100 & 0 & 0 \\
Estatura & 45 & 98 & 1 & 2 \\
Perímetro cefálico & 41 & 89 & 5 & 11 \\
Orientações às mães/responsáveis & 41 & 89 & 5 & 11 \\
Desenvolvimento neuropsicomotor & 12 & 26 & 34 & 74 \\
\hline
\end{tabular}

Com relação aos dados antropométricos, observou-se que 46 (100\%) das crianças foram pesadas, já a estatura foi verificada em 41 (89\%) das crianças avaliadas durante a consulta de puericultura. Ressalta-se que a literatura afirma que o peso e a estatura refletem o crescimento infantil. ${ }^{6,11}$

0 crescimento é considerado o aumento do tamanho corporal, sendo avaliado por meio do peso e crescimento linear; e, ainda, é um processo biológico, de multiplicação e aumento do tamanho celular. É considerado, também, como um dos melhores indicadores de saúde da criança, em razão de sua estreita dependência de fatores ambientais, tais como alimentação, ocorrência de doenças, cuidados gerais e de higiene, condições de habitação e saneamento básico, acesso aos serviços de saúde, refletindo assim, as condições de vida da criança, no passado e no presente. ${ }^{6}$ 


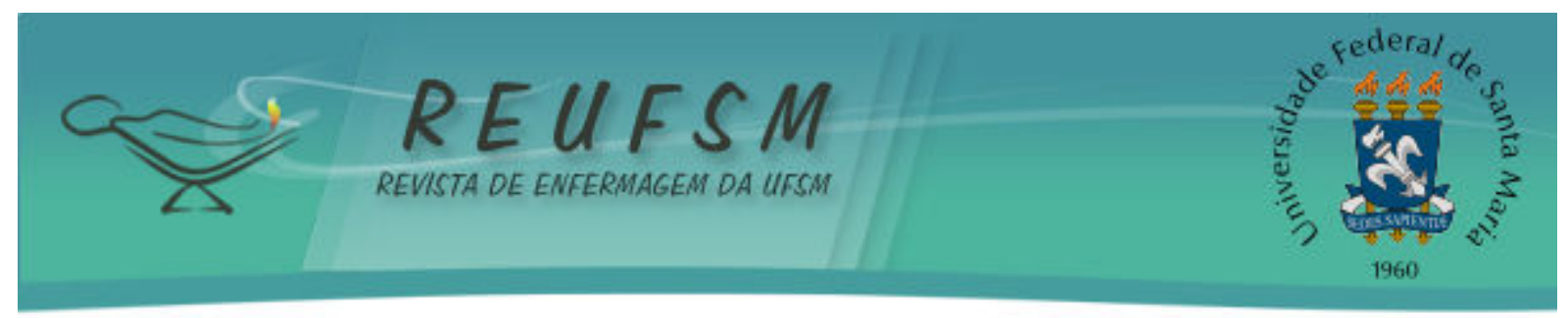

No concernente ao perímetro cefálico, outro dado que deve ser aferido na consulta, verificou-se na população estudada que 41 (89\%) das consultas registraram esse dado. 0 perímetro cefálico é uma variável que avalia o crescimento da cabeça/cérebro de crianças nos dois primeiros anos de vida. No caso desse índice apresentar desvios da faixa considerada de normalidade (conforme o gráfico disponível na Caderneta da Criança), a criança poderá ser encaminhada a um especialista ou a uma equipe multiprofissional, no intuito de afastar diagnóstico de microcefalia ou de macrocefalia. ${ }^{6}$

Já os perímetros torácico e abdominal não são registrados nos prontuários, caracterizando que o enfermeiro não afere tais medidas, ou não as registram, revelando-se que não há valorização desse dado pelos profissionais em estudo.

Os perímetros cefálico, torácico e abdominal também precisam ser verificados em todas as consultas de puericultura. Os valores desses dados deverão ser sempre analisados em função da idade e do sexo da criança, pois são os principais determinantes de sua evolução. Embora sejam procedimentos simples, devem ser realizados cuidadosamente, com padronização. 0 monitoramento pleno do crescimento infantil deve ser feito após o nascimento, bem como a avaliação dos perímetros cefálico e torácico deve ser feito de modo longitudinal, utilizando-se um padrão como referencial de avaliação. ${ }^{12}$

As medidas antropométricas são fundamentais para o acompanhamento do crescimento e do desenvolvimento infantil, sendo que sua verificação periódica é imprescindível para que ocorra a identificação precoce de alterações e sejam realizadas intervenções mais precisas e eficazes, garantindo assim qualidade de vida e saúde da criança.

Salienta-se que o Ministério da Saúde recomenda que os serviços de saúde realizem esforços para possuir os equipamentos necessários e os recursos humanos capacitados para interpretar adequadamente os valores antropométricos obtidos durante a consulta de puericultura, de modo que saibam orientar satisfatoriamente os pais acerca desses achados. ${ }^{6}$

No tocante aos marcos do DNPM, verificou-se apenas um registro (2\%). Igualmente, verificaram-se escassas as orientações sobre cuidados que contribuem para a aquisição de habilidades esperadas de acordo com o período avaliado, além de inexistir um roteiro utilizado pelo enfermeiro durante a evolução da consulta. 0 acompanhamento do DNPM é de fundamental importância na puericultura, pois tem o objetivo de detectar precocemente atrasos e deficiências, para que intervenções e encaminhamentos sejam realizados a tempo, evitando comprometimento no desenvolvimento infantil.

Sendo assim, infere-se que falhas no planejamento das ações em saúde, por parte dos enfermeiros, podem impactar na saúde da população infantil. Ainda, de acordo com o Ministério da Saúde, o acompanhamento do DNPM deve compor o prontuário da criança e ser utilizado em todas as consultas, a fim de detectar qualquer alteração no desenvolvimento normal, para que se possa oferecer orientações e agilizar encaminhamentos o mais célere possível. ${ }^{6}$

Também, observou-se que na maioria dos prontuários (98\%) estava registrado o tipo de alimentação oferecida à criança, dado relevante, já que a partir dessa informação o enfermeiro tem maiores condições de orientar os pais/responsáveis com relação à alimentação mais adequada para a idade.

Outra questão de vital importância que deve ser observada durante a puericultura diz respeito à imunização. Notou-se que em 100\% dos prontuários analisados não constavam esse registro, o que demonstra que o enfermeiro pode não observar o calendário de vacinação da criança ou simplesmente não registrar na consulta realizada. Destaca-se que informações não registradas são ações realizadas que dificilmente podem ser respaldadas. A imunização constitui uma das principais linhas de cuidado que deve ser monitorada pela equipe de saúde, promovendo busca-ativa imediata dos faltosos. ${ }^{3}$ 


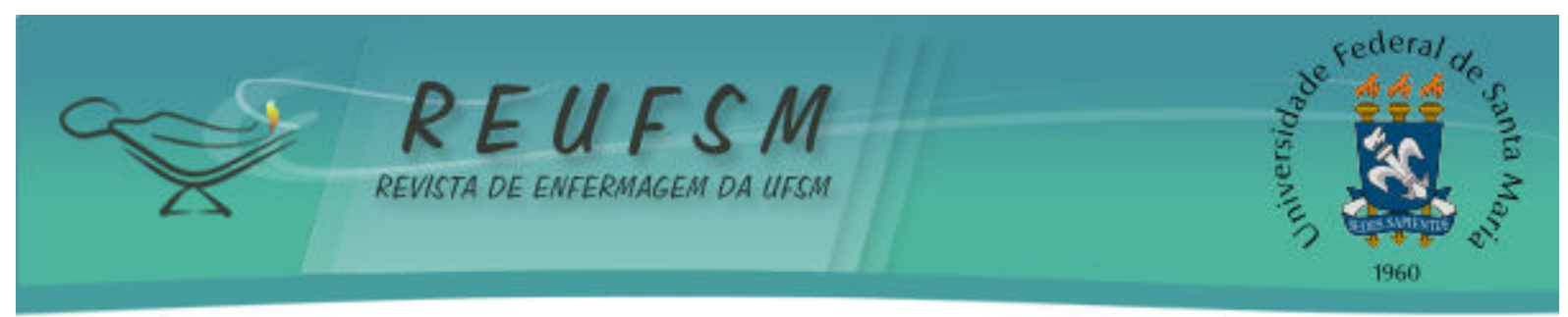

Os registros de enfermagem são de suma importância durante a assistência aos usuários, de modo que a inexistência de registro de atendimento é uma falha constante da classe, o que desvaloriza o trabalho do enfermeiro, visto que o que não é registrado, não pode ser computado como realizado. A qualidade dos registros feitos pelos enfermeiros favorece o julgamento clínico e a aplicação do processo de enfermagem, reforça o papel do profissional e contribui para a sistematização da sua prática. ${ }^{13}$

0 enfermeiro na consulta de puericultura deve utilizar os registros em gráficos de avaliação e comparação; observar o desenvolvimento, o ganho ponderal e o de estatura; fazer - levantamento do estado de saúde da criança; além de clarificar as necessidades e preocupações dos pais. O registro dessas informações é fundamental para identificar a situação singular de cada criança, possibilitando-lhe a prescrição do cuidar de enfermagem. ${ }^{8}$

Não obstante, é importante trazer a discussão que juntamente à aferição e ao registro adequado das medidas antropométricas a consulta de enfermagem deve privilegiar o diálogo e a orientação prestada às mães e às famílias. Estudo feito em um município do Paraná aponta que a puericultura realizada por enfermeiros não se restringe a pesagem e verificação de medidas antropométricas, mas sim possui ênfase na orientação acerca de questões relacionadas à saúde da criança. ${ }^{14}$

Salienta-se que a educação em saúde é indispensável no trabalho do enfermeiro em puericultura, já que na ESF esta, assim como as demais ações, deve ser centrada na família e entendida a partir do seu ambiente físico e social, enfatizando uma compreensão ampliada do processo saúde/doença e da necessidade de intervenções para além das práticas curativas. A educação em saúde também tenta buscar e ampliar a autonomia da mãe e reforçar sua condição de sujeito social, a fim de torná-la capaz de prestar o melhor cuidado ao seu filho. Entretanto, os registros nos prontuários estudados revelam uma atenção incipiente a essa prática ${ }^{14}$, pois as informações registradas no prontuário foram sobre amamentação, eliminações, vacinação, higiene, porém com superficialidade e escassez de detalhes.

Há décadas a enfermagem utiliza a sistematização da assistência (SAE), baseada no método científico para descrever, explicar e predizer seu exercício e seus resultados. ${ }^{15}$ Assim, esta tem a finalidade de validar a prática e dar à sociedade uma visão da profissão que fomente sua autonomia enquanto classe profissional. Para tanto, faz uso de habilidades cognitivas, técnicas e interpessoais, satisfazendo as necessidades do usuário e da família.

Os dados coletados permitiram observar que, na unidade pesquisada, não há emprego da SAE na realização da puericultura. A consulta de enfermagem de forma sistematizada auxilia no atendimento integral e na abordagem individualizada, executando e avaliando ações para a promoção, a proteção, a recuperação e a reabilitação da saúde. ${ }^{16}$

A SAE pode contribuir com os objetivos da ESF, pois os profissionais devem ter competências e habilidades que auxiliem na identificação dos mais variados problemas de saúde e fazer com que as soluções buscadas proporcionem um atendimento de qualidade. ${ }^{11}$

Os dados encontrados no presente estudo, com relação à falta de sistematização do atendimento realizado na puericultura e a ausência de registro de enfermagem nos prontuários das crianças atendidas, corroboram, em parte, com os resultados encontrados em outros estudos. ${ }^{11,17}$ Tais pesquisas apontam que o acompanhamento realizado à criança não seguia a lógica das políticas públicas de atenção à saúde da criança preconizadas pelo Ministério da Saúde.

Por fim, para discutir as potencialidades e limitações da puericultura, realizou-se uma breve reflexão, a partir dos dados encontrados e da literatura que aborda o tema, a qual permite traçar algumas considerações.

As potencialidades que a puericultura pode atingir são amplas, como a redução da morbimortalidade infantil por meio do aumento da cobertura vacinal, o estabelecimento 


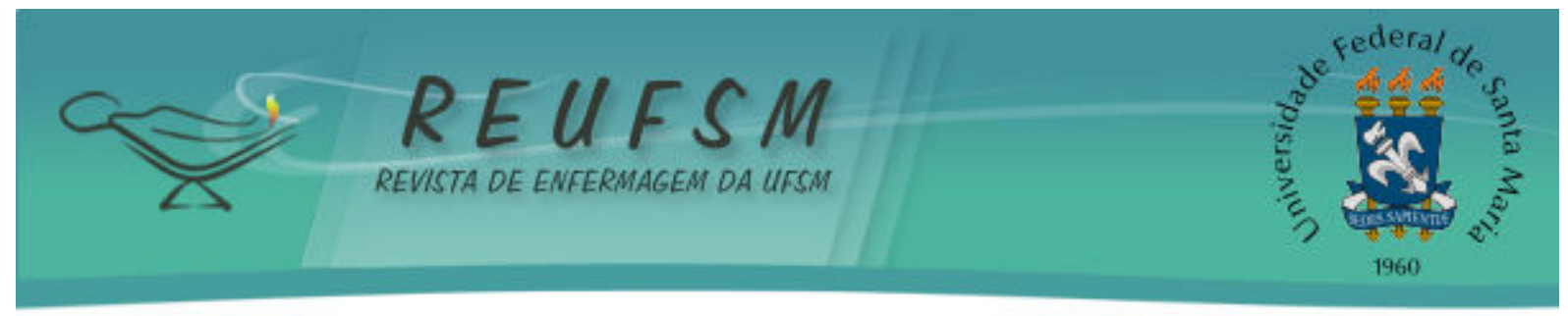

de vínculos com as famílias, a detecção precoce de agravos e encaminhamentos, a amplitude de orientações gerais as mães. ${ }^{6}$

Além disso, é feita educação em saúde quanto cuidados básicos de higiene, alimentação, eliminações, sono, prevenção de violência e acidentes da infância, práticas essas preconizadas pelo Ministério da Saúde $^{6}$ as quais não foram identificadas em sua integralidade no presente estudo. Destaca-se que estas são ações de promoção à saúde e prevenção de agravos que certamente trarão a melhoria da qualidade de vida da população infanti ${ }^{1-2,4}$.

Contudo, ao realizar essa reflexão, foi possível identificar, por meio dos registros analisados, algumas limitações presentes na prática assistencial do enfermeiro, que podem interferir diretamente na qualidade da puericultura, tais como: inexistência de sistematização do atendimento; assistência com foco curativo; irregularidade na frequência dos atendimentos; registros inconsistentes e/ou ausentes sobre as ações realizadas, com ênfase no peso e estatura; ausência do acompanhamento do DNPM.

Nesse âmbito, a literatura aponta alguns fatores que interferem na realização da puericultura. Sobre os usuários, há um estudo o qual aponta que a estrutura familiar não nuclear, ter mais de um filho, a falta de conscientização sobre a importância da puericultura, a insatisfação com o serviço de saúde e a falta de acessibilidade, são aspectos que dificultam a realização da puericultura ${ }^{18}$. Tais dados poderiam explicar, em parte, o início tardio e/ou a irregularidade no atendimento às crianças.

Em relação aos fatores que envolvem o trabalho do enfermeiro, há estudos os quais mostram dados semelhantes ao presente, sendo evidenciado que o enfermeiro realiza a puericultura de maneira fragmentada e esporádica, restringindo-se às medidas de peso e altura e ao atendimento à demanda espontânea. ${ }^{4,19}$ Além disso, a literatura expõe que a falta de planejamento, pelo enfermeiro, para acompanhamento periódico das crianças, a sobrecarga de trabalho e o processo de trabalho da equipe de enfermagem, com prioridade para ações curativas, são fatores que interferem na qualidade da puericultura. ${ }^{4}$

Ressalta-se que a puericultura proporciona um cuidado na perspectiva da integralidade, assim, a atuação do enfermeiro precisa estar em consonância com as políticas públicas. Para tanto, o enfermeiro, junto com a equipe de saúde, necessitam de constante avaliação e aprimoramento de seu processo de trabalho. ${ }^{4}$

\section{CONCLUSÃO}

No tocante à pesquisa realizada, percebe-se uma realidade preocupante, pois se observa valorização de registros sobre medidas antropométricas e poucos, até mesmo ausentes, registros sobre ações educativas em saúde. Os resultados apontam que o serviço de saúde deve ser reorganizado, pautando suas ações no conceito de promoção à saúde infantil.

Com o presente estudo foi possível perceber que o atendimento de puericultura não está sistematizado, tendo em vista o atendimento com frequência irregular. Salientase que o atendimento se dá por demanda espontânea, o que favorece mais a prática curativa do que a promoção da saúde e prevenção de agravos. Existem, também, falhas consideráveis no registro em prontuário por parte do enfermeiro, pois provavelmente muitas ações e orientações realizadas durante a consulta foram consideradas, porém não comprovadas pelo fato de não estarem registradas. Tem-se ainda que não há registro da avaliação do DNPM e da imunização, dados estes de fundamental importância para que intervenções sejam realizadas em tempo hábil, evitando agravamento na saúde e desenvolvimento da criança.

Sobre as potencialidades e limitações, o estudo adverte que os enfermeiros devem repensar acerca de sua prática em puericultura constantemente. Ao visualizar e valorizar 


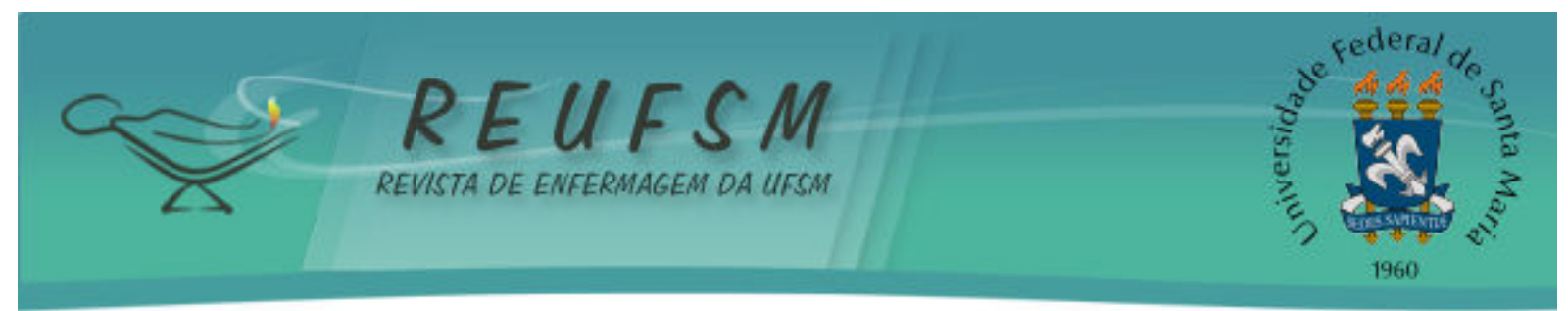

as potencialidades da puericultura, os profissionais podem adquirir uma postura pró-ativa, segura e confiante no seu trabalho, podendo alcançar ótimos resultados, visando evitar a perda de oportunidade de atuação. Além de repensar sobre as limitações, o enfermeiro pode juntamente com a equipe procurar medidas que visem evitá-las e/ou superá-las no sentido de acreditar no trabalho e desenvolvê-lo de maneira comprometida e responsável para com a saúde das crianças.

Sendo assim, este estudo serve de subsídio para que os gestores e profissionais do município se conscientizem acerca da necessidade de melhorias na assistência à saúde oferecida à criança. Estudos dessa natureza são relevantes, a fim de detectar possíveis falhas na assistência à saúde da criança, viabilizando oportunidades de mudanças, para que de fato o modelo assistencial em vigência seja efetivado.

\section{REFERÊNCIAS}

1. Costa L, Silva EF, Lorenzini E, Strapasson MR, Pruss ACSF, Bonilha ALL. Significado da consulta de enfermagem em puericultura: percepção de enfermeiras de estratégia saúde da família. Ciênc Cuid Saúde. 2012;11(4):792-8.

2. Leite GB, Bercini LO. Caracterização das crianças atendidas na puericultura do Programa Saúde da Família do Município de Campo Mourão, Paraná, em 2003. Ciênc Cuid Saúde. 2005;4(3):224-30.

3. Brasil. Ministério da Saúde. Secretaria de Atenção à Saúde. Departamento de Ações Programáticas Estratégicas. Agenda de compromissos para a saúde integral da criança e redução de mortalidade infantil. Brasília (DF): Ministério da Saúde; 2004.

4. Assis WD, Collet N, Reichert APS, Sá LD. Processo de trabalho da enfermeira que atua em puericultura nas unidades de saúde da família. Rev Bras Enferm. 2011;64(1):38-46.

5. Piccini RX, Facchini LA, Tomasi E, Thumé E, Silveira DS, Siqueira FV, et al. Efetividade da atenção pré-natal e de puericultura em unidades básicas de saúde do Sul e do Nordeste do Brasil. Rev Bras Saúde Matern Infant. 2007;7(1):75-82.

6. Brasil. Ministério da Saúde. Secretaria de Políticas de Saúde. Departamento de Atenção Básica. Saúde da criança: crescimento e desenvolvimento infantil. Brasília: Ministério da Saúde; 2012. (Cadernos de Educação Básica; 33).

7. Vasconcelos VM, Frota MA, Martins MC, Machado MMT. Puericultura em Enfermagem e educação em saúde: percepção das mães na Estratégia Saúde da Família. Esc Anna Nery Rev Enferm. 2012,16(2):326-31.

8. Oliveira VC, Cadete MMM. A consulta de enfermagem no acompanhamento do crescimento e desenvolvimento infantil. REME Rev Min Enferm. 2007;11(1):77-80.

9. Dyniewicz AM. Métodos e técnicas de pesquisa para iniciantes. São Caetano do Sul: Difusão; 2007.

10. Brasil. Ministério da Saúde. Secretaria de Atenção à Saúde. Departamento de Ações Programáticas e Estratégicas. Atenção à saúde do recém-nascido: guia para os profissionais de saúde. Brasília: Ministério da Saúde; 2011. (Série A. Normas e Manuais Técnicos).

11. Lima GGT, Silva MFOC, Costa TNA, Neves AFGB, Dantas RA, Lima ARSO. Registros do enfermeiro no acompanhamento do crescimento e desenvolvimento: enfoque na consulta de puericultura. Rev RENE. 2009;10(3):117-24. 


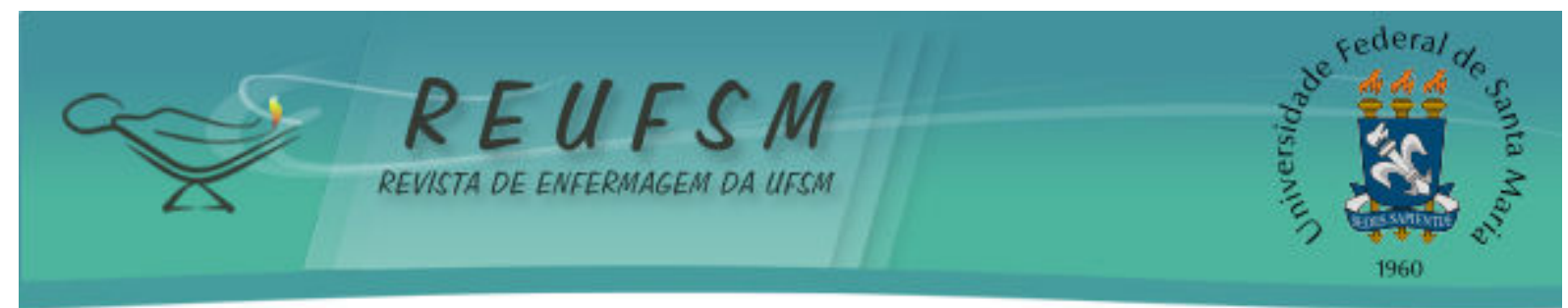

12. Jaldin MGM, Pinheiro FS, Santos AM, Muniz NC, Brito LMO. Crescimento do perímetro cefálico nos primeiros seis meses em crianças em aleitamento materno exclusivo. Rev Paul Pediatr. 2011;29(4):509-14.

13. Fernández-Sola C, Granero-Molina J, Mollinedo-Mallea J, Gonzales MHP, AguileraManrique G, Ponce ML. Desarrollo y validación de un instrumento para la evaluación inicial de enfermeira. Rev Esc Enferm USP. 2012;46(6):1415-22.

14. Vieira VCL, Fernandes CA, Demitto MO, Bercini LO, Scochi MJ, Marcon SS. Puericultura na atenção primária à saúde: atuação do enfermeiro. Cogitare Enferm. 2012;17(1):119-25.

15. Cavalcante RB, Otoni A, Bernardes MFVG, Cunha SGS, Santos CS, Silva PC. Experiências de sistematização da assistência de enfermagem no Brasil: um estudo bibliográfico. Rev Enferm UFSM [Internet]. 2011 abr [acesso em 2013 abr 3];1(3):461-71. Disponível em: http://cascavel.ufsm.br/revistas/ojs-2.2.2/index.php/reufsm/article/view/2832/2396.

16. Campos RMC, Ribeiro CA, Silva CV, Saparolli ECL. Consulta de enfermagem em puericultura: a vivência do enfermeiro na Estratégia de Saúde da Família. Rev Esc Enferm USP. 2011;45(3):566-74.

17. Ceia MLM, Cesar JA. Avaliação do preenchimento dos registros de puericultura em unidades básicas de saúde em Pelotas, RS. Rev AMRIGS. 2011;55(3):244-9.

18. Vitolo MR, Gama CM, Campagnolo PDB. Frequency of public child care service use and associated factors. J Pediatr (Rio J). 2010;86(1):80-4.

19. Moita KMT, Queiroz MVO. Puericultura: concepções e prática do enfermeiro no Programa de Saúde da Família. Rev RENE. 2005;6(1):9-19.

Data de recebimento: 04/04/2013

Data de aceite: 16/05/2014

Contato com autor responsável: Tatiane Baratieri

Endereço postal: Rua Simeão Camargo Varela de Sá, nº 03. Bairro Vila Carli. Guarapuava/PR.

CEP: $85040-080$

E-mail: baratieri.tatiane@gmail.com 